\title{
Papers
}

\section{Postcards from the EDge project: randomised controlled trial of an intervention using postcards to reduce repetition of hospital treated deliberate self poisoning}

Gregory L Carter, Kerrie Clover, Ian M Whyte, Andrew H Dawson, Catherine D’Este

\begin{abstract}
Objective To determine whether an intervention using postcards (postcards from the EDge project) reduces repetitions of hospital treated deliberate self poisoning.

Design Randomised controlled trial.

Setting Regional referral service for general hospital treated deliberate self poisoning in Newcastle, Australia.

Participants 772 patients aged over 16 years with deliberate self poisoning.

Intervention Non-obligatory intervention using eight postcards over 12 months along with standard treatment compared with standard treatment alone.

Main outcome measures Proportion of patients with one or more repeat episodes of deliberate self poisoning and the number of repeat episodes for deliberate self poisoning per person in 12 months.

Results The proportion of repeaters with deliberate self poisoning in the intervention group did not differ significantly from that in the control group $(57 / 378,15.1 \%, 95 \%$ confidence interval $11.5 \%$ to $18.7 \%$ v $68 / 394,17.3 \%, 13.5 \%$ to $21.0 \%$ : difference between groups $-2 \%,-7 \%$ to $3 \%$ ). In unadjusted analysis the number of repetitions were significantly reduced (incidence risk ratio $0.55,0.35$ to 0.87 ).

Conclusion A postcard intervention reduced repetitions of deliberate self poisoning, although it did not significantly reduce the proportion of individual repeaters.
\end{abstract}

\section{Introduction}

Deliberate self poisoning is common, accounting for $5 \%$ of admissions to general hospitals in Australia. ${ }^{1}$ In the United Kingdom, deliberate self harm is one of the top five reasons for acute medical admissions. ${ }^{2}$ Most patients with deliberate self harm have initial contact with hospital through the emergency department. Repetition of deliberate self harm is also common, ranging from $6 \%$ to $30 \%$ in 12 months. ${ }^{2}$ Repetition is strongly associated with subsequent suicide and has important implications for healthcare resources. Three non-pharmacological interventions were effective in reducing repetition in selected subsets of populations with deliberate self harm, ${ }^{3-5}$ but interventions are needed that could be economically delivered to the entire population of patients who deliberately self harm.

We tested the efficacy of a postcard intervention (postcards from the EDge project) over 12 months on repetition of hospital treated deliberate self poisoning.

\section{Methods}

The Hunter Area Toxicology Service is a regional toxicology unit at the Newcastle Mater Hospital, New South Wales, Australia, serving a primary referral population of 385000 adults and a tertiary referral population of a further 170000 . All patients presenting with poisoning to emergency departments in the greater Newcastle region are admitted to the service or notified to the service and entered prospectively into a clinical database. ${ }^{6}$ The psychiatry department of the Newcastle Mater Hospital sees all patients with deliberate self poisoning for assessment and diagnosis and to determine discharge destination and follow-up. Details of the model of service for these patients have been described. $^{\text {? }}$

Potentially eligible participants were those aged over 16 years who presented to the toxicology service with deliberate self poisoning during April 1998 to December 2001. We excluded patients incapable of informed consent, those of no fixed address, those with insufficient English to complete a structured interview, and those who posed a potential threat to an interviewer.

\section{Variables}

Our two dependent variables were the proportion of patients with at least one repeat episode of deliberate self poisoning in 12 months and the number of repeat episodes of deliberate self poisoning per individual over 12 months.

We extracted the descriptive variables of the sample from the toxicology service's database, which were derived from the standardised clinical assessment of patients with deliberate self poisoning. ${ }^{78}$ The categorical variables were sex, marital status (married or de facto married versus never married, separated, divorced, or widowed), employment (full time or part time versus unemployed, pensioner, student, or other), admission to an intensive care unit, time of admission (9 am to 5 pm weekdays versus out of hours), and discharge destination (psychiatric hospital versus all others). The continuous variables were age, length of stay in hours, median number of previous admissions to the toxicology service for deliberate self poisoning, and number of psychiatric diagnoses from clinical assessment.

\section{Design and randomisation}

We used a randomised consent design, using the single consent version (Zelen design). ${ }^{9}{ }^{10}$ This design is a variation on the standard randomised controlled experimental design, where partici-

The postcard is on bmj.com 
pants are randomised to control or intervention before consent is sought. In the single consent version, written informed consent to receive the intervention (eight non-obligatory postcards) was sought from participants randomised to the intervention. We assessed the outcomes by an intention to treat analysis on the basis of allocation.

Randomisation was by database (HanDBase version 2.0; DDH Software, FL, USA) on a personal digital assistant (Palm III; Palm, CA, USA) that was populated with a pregenerated randomisation schedule (in blocks of 10) and carried by the duty toxicologist. To avoid recruiting patients more than once, the duty toxicologist sought information on identification in this database and entered only new patients. To maintain blinding to allocated group during recruitment, randomisation was not revealed until after all information was entered and eligibility had been determined. The allocation group was then revealed to obtain patient consent. To monitor any potential alterations (interference), we kept a duplicate record in a hidden field of the database and a copy was held on a separate computer for later verification of correct allocation. All other clinical and research staff remained blinded to allocation.

\section{Intervention}

Our intervention was based on a previous study, which showed significantly reduced death by suicide in a population of psychiatric inpatients who received long term contact by letter after hospital discharge. ${ }^{11}{ }^{12}$ Our intervention comprised a postcard sent to participants in a sealed envelope at 1, 2, 3, 4, 6, 8, 10, and 12 months after discharge (see postcard on bmj.com).

\section{Sample sizes}

We calculated several sample sizes for different estimates of possible effects. ${ }^{13}$ We determined that to detect a difference in proportions $(\mathrm{P}=0.05,80 \%$ power) of $15 \%$ to $10 \%$ required 1364 participants, a difference of $20 \%$ to $10 \%$ required 392 , and a difference of $30 \%$ to $20 \%$ required 293 .

On the basis of clinical experience and previous research we anticipated that between $15 \%$ and $30 \%$ of participants in the control group would repeat deliberate self poisoning within 12 months, with an average of two episodes, giving overall rates for deliberate self poisoning of 0.30 to 0.60 . A sample of 400 participants in each group would allow detection of absolute differences between groups of 0.10 to $0.15(\mathrm{P}=0.05,80 \%$ power $)$ and 0.12 to 0.17 ( $\mathrm{P}=0.05,90 \%$ power) yielding relative risks of 0.67 to 0.75 and 0.60 to 0.72 for $80 \%$ and $90 \%$ power, respectively. This sample size would be adequate to detect differences in the proportion of participants with any deliberate self poisoning of $7-9 \%$ (80\% power) and $8-10 \%(90 \%$ power and $5 \%$ significance level), which we considered would represent a clinically significant reduction.

\section{Statistical analyses}

We analysed data using SPSS version 10.0 and Intercooled Stata 7. We used the $\chi^{2}$ test to determine the difference in proportions of participants with any repetition of deliberate self poisoning. We initially considered a Poisson model for the number of repeat episodes of deliberate self poisoning per individual; however, exploratory analysis indicated that the variance of the number of deliberate self poisonings was much greater than the mean. We therefore used the negative binomial model. We compared the risk of deliberate self poisoning events per individual in the intervention group with that in the control group using negative binomial regression, reported as the incidence risk ratio with $95 \%$ confidence intervals. As we found a difference between the sexes, we undertook subgroups analyses of treatment effect for men and women, using negative binomial models.

Twenty participants in the control group received the intervention due to clerical errors but were included in the control group for the intention to treat analyses.

\section{Results}

We excluded 150 of 922 patients (16\%) assessed for eligibility to our study, leaving 772 participants-394 in the control group and 378 in the intervention group (fig 1). Among the intervention group, 76 refused the intervention, one missed the intervention, and 32 did not receive the full intervention, usually because the participants were reported as unknown at the address on the postcards. Table 1 shows the characteristics of the participants at baseline. Overall, 129 of 772 (17\%) participants had a previous episode of hospital treated deliberate self poisoning: 66 of $394(17 \%)$ in the control group and 63 of $378(17 \%)$ in the intervention group. Poisonings were classified as pharmaceuticals only $(473,61 \%)$, pharmaceuticals plus alcohol $(217,28 \%)$, opioid or amphetamine (20,3\%), carbon monoxide (17, $2 \%)$, herbicide or rodenticide $(11,1 \%)$, insulin $(7,1 \%)$, any deliberate self harm with deliberate self poisoning (23, 3\%), and unknown $(4,1 \%)$.

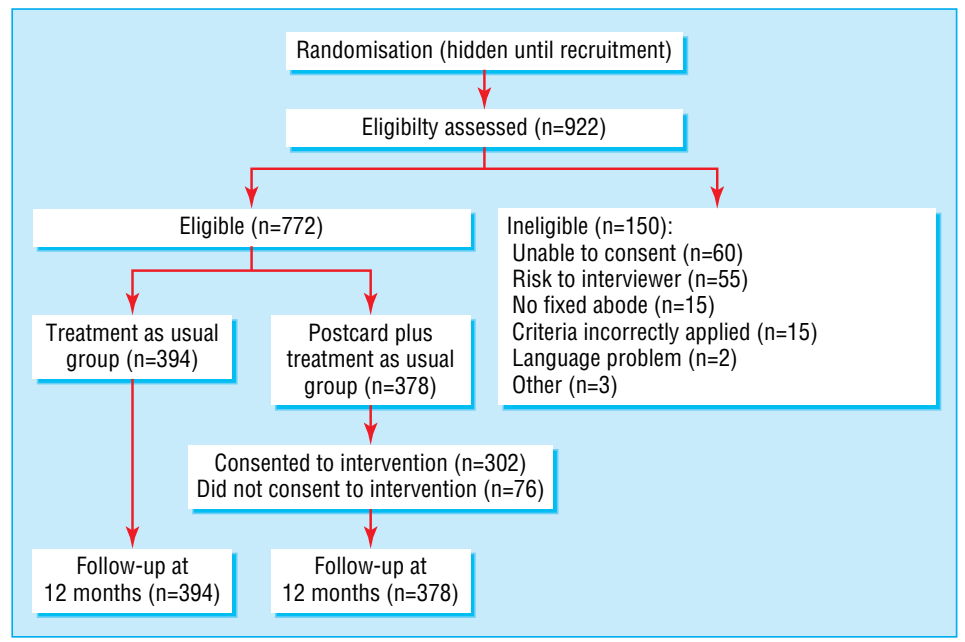

Fig 1 Flow of participants through trial 
Table 1 Characteristics of groups receiving a postcard intervention or standard care for hospital treated deliberate self poisoning. Values are numbers (percentages) unless stated otherwise

\begin{tabular}{|c|c|c|c|c|}
\hline Characteristic & Missing data & Total $(\mathrm{n}=772)$ & Control group $(\mathrm{n}=394)$ & Postcard group ( $\mathrm{n}=378)$ \\
\hline Women & 1 & $524(68)$ & $291(74)$ & $233(62)$ \\
\hline Married or de facto married & 31 & $256(35)$ & $118(31)$ & $138(38)$ \\
\hline Employed & 153 & $162(26)$ & $88(27)$ & 74 (26) \\
\hline Admitted to intensive care & 0 & $113(15)$ & $60(15)$ & $53(14)$ \\
\hline Admitted outside office hours & 0 & $581(75)$ & $296(75)$ & $285(75)$ \\
\hline Discharged to psychiatric hospital & 2 & $208(27)$ & $106(27)$ & $102(27)$ \\
\hline $\begin{array}{l}\text { Previous admission for deliberate self } \\
\text { poisoning }\end{array}$ & 0 & $129(17)$ & $66(17)$ & $63(17)$ \\
\hline Median (interquartile range) age (years) & 0 & $33(24-44)$ & $34(23-45)$ & $33(24-42)$ \\
\hline $\begin{array}{l}\text { Median (interquartile range) length of stay } \\
\text { (hours) }\end{array}$ & 0 & $18(12-30)$ & $18(13-31)$ & $17(12-29)$ \\
\hline $\begin{array}{l}\text { Median No of previous admissions for } \\
\text { deliberate self poisoning (interquartile } \\
\text { range) }\end{array}$ & 0 & $0(0-0)$ & $0(0-0)$ & $0(0-0)$ \\
\hline $\begin{array}{l}\text { Median No of psychiatric diagnoses } \\
\text { (interquartile range) }\end{array}$ & 0 & $2(1-2)$ & $2(1-3)$ & $2(1-3)$ \\
\hline
\end{tabular}

\section{Proportion of participants repeating deliberate self} poisoning

Fifty seven of the participants in the intervention group $(15.1 \%$, $95 \%$ confidence interval $11.5 \%$ to $18.7 \%$ ) had one or more

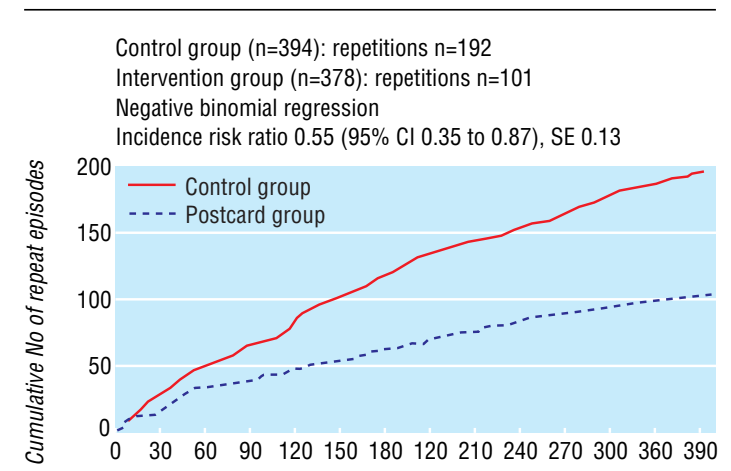

Days

Fig 2 Cumulative number of repeat episodes of hospital treated deliberate self poisoning repeat episodes of deliberate self poisoning compared with 68 $(17.3 \%, 13.5 \%$ to $21 \%)$ in the control group (difference between groups $-2 \%,-7 \%$ to $3 \%$ ), with no significant difference $\left(\chi^{2}=0.675, \mathrm{df}=1, \mathrm{P}=0.41\right)$.

\section{Number of repeat episodes}

The cumulative number of repeat episodes of deliberate self poisoning was 192 in the control group and 101 in the intervention group (fig 2).

The risk of repetition was statistically significantly lower in the intervention group than in the control group (incidence risk ratio $0.55,95 \%$ confidence interval 0.35 to 0.87 ; table 2). Although we found no significant interaction between treatment and sex, subgroup analyses by sex showed that the treatment was effective for women $(0.54,0.30$ to 0.96$)$ but not for men $(0.97$, 0.48 to 2.0). Table 3 shows the number of repeat episodes by allocated group and sex.

\section{Discussion}

A simple intervention comprising a postcard sent to patients at intervals after discharge for an episode of deliberate self poison-

Table 2 Negative binomial models for intervention effect on repetitions of deliberate self poisoning and for subgroup analyses by sex

\begin{tabular}{|c|c|c|c|c|}
\hline & Incidence risk ratio & SE $(95 \% \mathrm{Cl})$ & $Z$ value ${ }^{*}$ & $P$ value \\
\hline \multicolumn{5}{|c|}{ Group (unadjusted) } \\
\hline Control & 1.00 & & & \\
\hline Postcards & 0.55 & $0.13(0.35$ to 0.87$)$ & -2.56 & 0.010 \\
\hline \multicolumn{5}{|c|}{ Subgroup analyses } \\
\hline \multicolumn{5}{|l|}{ Men $(n=247)$ : } \\
\hline Control & 1.00 & & & \\
\hline Postcards & 0.97 & 0.35 (0.48 to 1.98) & -0.08 & 0.937 \\
\hline \multicolumn{5}{|c|}{ Women $(n=524)$ : } \\
\hline Control & 1.00 & & & \\
\hline Postcards & 0.54 & 0.16 (0.30 to 0.96$)$ & -2.09 & 0.037 \\
\hline
\end{tabular}

Table 3 Number of repeat episodes of deliberate self poisoning by sex and by intervention group. Values are numbers (percentages)

Control group ( $\mathrm{n}=394)$

\begin{tabular}{|c|c|c|c|c|c|c|c|c|}
\hline \multirow[b]{2}{*}{ No of repetitions } & & \\
\hline & Men $(n=102)$ & Total repetitions & $\begin{array}{l}\text { Women } \\
(\mathrm{n}=291)\end{array}$ & Total repetitions & Men $(n=145)$ & Total repetitions & $\begin{array}{l}\text { Women } \\
(n=233)\end{array}$ & Total repetitions \\
\hline$\overline{0}$ & 86 (84) & 0 & $240(83)$ & 0 & $125(86)$ & 0 & $196(84)$ & 0 \\
\hline 1 & $13(13)$ & 13 & $25(9)$ & 25 & $13(9)$ & 13 & 21 (9) & 21 \\
\hline 2 & $2(2)$ & 4 & $8(3)$ & 16 & $5(3)$ & 10 & $10(4)$ & 20 \\
\hline$\geq 3$ & $1(1)$ & 4 & $18(6)$ & 125 & 2 (1) & 6 & $6(3)$ & 31 \\
\hline
\end{tabular}

One control participant with missing data for sex had five readmissions. 
ing nearly halved the number of repeat episodes in 12 months. Although the intervention did not reduce the proportion of individual repeaters, it did reduce the number of events per patient.

\section{Strengths and weaknesses of the study}

Our study had several strengths. We used a randomised consent design, which was suited to this study and clinical population. The characteristics of the patients at baseline and principal outcomes were tracked by a database, allowing for complete followup. This method has not been used previously in testing interventions for suicidal behaviours. The statistical, ethical, and practical issues concerning the use of a randomised consent design have been extensively reviewed ${ }^{14}{ }^{15}$ and, despite some of the difficulties, this design has been used in recently published studies. ${ }^{16} 17$

The quality of the randomisation was good. Randomisation was undertaken using a handheld personal computer in combination with another computer that would detect any errors of allocation status and interference with randomisation.

All participants and clinical and research staff were blind to outcome, with data extraction done five months after completion of the study period. Only the recruiting toxicologists and secretary responsible for managing the mailing database and postcards were not blind to the allocation status.

Our study had some limitations and caution is needed when interpreting the results. Fewer than $20 \%$ of the participants had any repeat episodes of deliberate self poisoning, and a subgroup of repeaters had a highly skewed pattern of more than one repeat episode. It is not known to what extent our population and model of clinical service ${ }^{7}$ would be generalisable to other settings. As we studied patients with deliberate self poisoning, the results cannot be generalised to patients with other forms of deliberate self harm.

Replications of this study and additional effectiveness trials would be necessary before considering widespread implementation. Our decision to include a subgroup analysis on the basis of sex was a retrospective one based on the findings from the primary outcomes. Caution is needed in interpreting such subgroup analyses because of (unplanned) reduced sample sizes, and judgment needs to be exercised regarding the biological plausibility of such analyses.

The estimate of efficacy of treatment effect may have been conservative for three reasons: 20 control participants were inadvertently exposed to the intervention, 76 intervention participants did not consent to receive the intervention, and 32 intervention participants were not exposed to the intervention.

\section{Implications of the study}

A simple postcard intervention nearly halved the number of repeat episodes for deliberate self poisoning within 12 months. The control group occupied hospital beds for 239 days (192 repetitions) compared with 129 days (101 repetitions) in the intervention group, a total of 110 bed days saved. This represented a considerable saving in opportunity costs, availability of hospital beds, and decrease in workload for the emergency department. The costs of stationery, post, maintenance of a mailing database, and staff time were estimated to be less than $\$ A 15$ (£6.25; \$11.52; €9.19) per participant. This low cost intervention seems to have substantial cost effectiveness. The simplicity of the intervention means that it could be delivered from hospitals that do not have extensive resources for patients with deliberate self poisoning.

The difference in total repetitions for deliberate self poisoning came from one main source; women with three or more repeat episodes (table 3), which accounted for a difference of 94 repeat episodes ( 125 by control participants and 31 by intervention participants). This was a surprising result as we expected that women multiple repeaters would be relatively unresponsive to such a simple intervention. Our low cost intervention can be applied to almost all adult patients with hospital treated deliberate self poisoning and can be used without identifying patients at high risk of repetition.

The mechanism of action for the postcard intervention is unknown and was not evaluated in this study. Nevertheless, the authors of a study using a letter based intervention ${ }^{12}$ speculated about increased social connectedness, a concrete expression that someone still cares about the patient. A similar interpretation would be reasonable for our study. Patients requiring hospital treatment for deliberate self poisoning may believe that their situation is hopeless, that no one cares about them, or that they are viewed as incompetent and undeserving of care. We do not know if the patients in this study experienced any degree of impaired social connectedness but they may well have done so. It may be that, when combined with the postcard intervention, a service model that emphasises respect for the patient, high quality medical and psychiatric management, and follow-up arrangements on discharge is able to reduce the feelings of lack of social connectedness. If so, then simply implementing a postcard intervention without the clinical model of care may not be as effective. The previous letter intervention ${ }^{12}$ was, however, effective, even for patients who eschewed all ongoing contact with treatment services, so this type of intervention may have independent effects on repetition of suicidal behaviour.

\section{Conclusion}

Previous studies of intervention to reduce repetition of deliberate self harm in unselected patient groups have been unsuccessful in reducing the proportion of repeaters. Even using a meta-analytic approach has shown no benefit from antidepressants, problem solving, intensive care with outreach, an emergency card, $^{18}$ a psychosocial crisis intervention, or guaranteed in-patient shelter in cases of emergency. ${ }^{19}$

Although we found no significant difference in the proportion of individual patients who repeated we did find that a low cost postcard intervention was effective in reducing the number of events per individual. The magnitude of the reduction, nearly $50 \%$, was clinically and statistically significant.

A small proportion of the deliberate self poisoning population accounts for substantial numbers of repetitions and treatment costs. This intervention produced considerable savings in opportunity costs for the hospital service in which it was tested. That the effect of reduction in repetitions was seen in only women suggests that future studies need to be adequately powered to test treatment effect by sex.

We thank Jerome Motto for his encouragement to use and modify the original intervention for this project; Trish McGettigan, Patrick Oakley, Nav Gupta, and Geoff Isbister for patient recruitment; Stuart Allen for data management support; Bob Goldney and Natalia Carter for alerting us to the Motto study; Julia Lowe and Dianne O'Connell for schooling us in Zelen designs; and Helen Rowsell, the departmental secretary, who managed the mailing database and mailing lists so diligently.

Contributors: GLC had the original idea for the study and did the original drafting of the manuscript. He is guarantor. GLC, IMW, and AHD planned the study. $\mathrm{KC}$ was responsible for managing the data sets and carrying out most of the analyses. CD'E undertook the negative binomial and other regression analyses and gave statistical advice on other issues. All authors reviewed the manuscript and replied to reviewers.

Funding: KC is funded by the NSW Health, Burdekin Mental Health Enhancement Strategy.

Competing interests: None declared. 


\section{What is already known on this topic}

Deliberate self harm is common and costly, with repetition rates of $6-30 \%$

Deliberate self poisoning is the commonest form of deliberate self harm

Few interventions effectively reduce repetitions of deliberate self harm

\section{What this study adds}

A simple, inexpensive, postcard intervention for patients with deliberate self poisoning reduced the number of events per individual, but did not reduce the proportion of individual repeaters

Ethical approval: This research project was approved by the Hunter Area Health Services research ethics committee (No 9710153.15).

1 McGrath J. A survey of deliberate self-poisoning. Med J Aust 1989;150:317-22.

2 House A, Owens D, Patchett L. Deliberate self-harm. Eff Health Care 1998;4:1-12.

3 Linehan MM, Armstrong HE, Suarez A, Allmon D, Heard HL. Cognitive-behavioral treatment of chronically parasuicidal borderline patients. Arch Gen Psychiatry 1991;48:1060-4.

4 Bateman A, Fonagy P. Effectiveness of partial hospitalization in the treatment of borderline personality disorder: a randomized controlled trial. Am J Psychiatry 1999;156:1563-9.

5 Guthrie E, Kapur N, Mackway-Jones K, Chew-Graham C, Moorey J, Mendel E, et al. Randomised controlled trial of brief psychological intervention after deliberate self poisoning. BMJ 2001;323:135-8.

6 Whyte IM, Buckley NA, Dawson AH. Data collection in clinical toxicology: are there too many variables? J Toxicol Clin Toxicol 2002;40:223-30.

7 Whyte IM, Dawson AH, Buckley NA, Carter GL, Levey CM. A model for the management of self-poisoning. Med J Aust 1997;167:142-6.

8 Buckley NA, Whyte IM, Dawson AH, Reith D. Preformatted admission charts for poisoning admissions facilitate clinical assessment and research. Ann Emerg Med 1999:34:476-82
9 Zelen M. A new design for randomized clinical trials. N Engl J Med 1979;300:1242-5.

10 Zelen M. Randomized consent designs for clinical trials: an update. Stat Med 1990;9:645-56.

11 Motto JA. Suicide prevention for high-risk persons who refuse treatment. Suicide Life Threat Behav 1976;6:223-30.

12 Motto JA, Bostrom AG. A randomized controlled trial of postcrisis suicide prevention. Psychiatric Serv 2001;52:828-33.

13 Carter G, Clover K, Fryer J. Deliberate self-harm: can we move the goal posts closer? eBMJ www.bmj.com/cgi/eletters/317/7156/44. 3-17-1999.

14 Torgerson DJ, Roland M. What is Zelen's design? BMJ 1998;316:606.

15 Altman DG, Whitehead J, Parmar MK, Stenning SP, Fayers PM, Machin D. Randomised consent designs in cancer clinical trials. Eur J Cancer 1995;31A:1934-44.

16 Van Buuren H, Rasch MC, Batenburg PL, Bolwerk CJ, Nicolai JJ, van der Werf SD, et al. Endoscopic sclerotherapy compared with no specific treatment for the primary prevention of bleeding from esophageal varices. A randomized controlled multicentre trial. BMC Gastroenterol 2003;3:22.

17 Turnbull DA, Wilkinson C, Gerard K, Shanahan M, Ryan P, Griffith EC, et al Clinical, psychosocial, and economic effects of antenatal day care for three medical complications of pregnancy: a randomised controlled trial of 395 women. Lancet 2004:363:1104-9.

18 Hawton K, Arensman E, Townsend E, Bremner S, Feldman E, Goldney R, et al. Deliberate self harm: systematic review of efficacy of psychosocial and pharmacological treatments in preventing repetition. BMJ 1998;317:441-7.

19 Van der Sande R, Buskens E, Allart E, Van der Graaf Y, Van Engeland H. Psychosocial intervention following suicide attempt: a systematic review of treatment interventions. Acta Psychiatr Scand 1997:96:43-50.

(Accepted 10 August 2005)

doi 10.1136/bmj.38579.455266.E0

Suicide Prevention Research Unit, Centre for Mental Health Studies, Faculty of Health, University of Newcastle, Newcastle, Australia

Gregory L Carter head

Kerrie Clover comjoint senior lecturer

Discipline of Clinical Pharmacology, Faculty of Health, University of Newcastle

Ian M Whyte conjoint professor

Andrew H Dawson conjoint professor

Centre for Clinical Epidemiology and Biostatistics, Faculty of Health, University of Newcastle, Australia

Catherine D'Este associate professor

Correspondence to: Dr Gregory Carter, Department of Consultation-Liaison

Psychiatry, Locked Bag No 7, Hunter Region Mail Centre, NSW 2310, Australia

gregory.carter@newcastle.edu.au 